\title{
Research Note \\ The effect of scattering on pulsar polarization angle
}

\author{
X. H. Li and J. L. Han \\ National Astronomical Observatories, Chinese Academy of Sciences, Jia-20 DaTun Road, Chaoyang District, Beijing 100012, \\ PR China \\ Received 2 May 2003 / Accepted 29 July 2003

\begin{abstract}
The low-frequency profiles of some pulsars manifest temporal broadening due to scattering, usually accompanied by flat polarization position angle (PA) curves. Assuming that the scattering works on the 4 Stokes parameters in the same way, we have simulated the effect of scattering on polarization profiles and find that the scattering can indeed flatten the PA curves. Since the higher-frequency profiles suffer less from scattering, they are convolved with scattering models to fit the observed low-frequency profiles. The calculated flat PA curves exactly reproduce the corresponding observations.
\end{abstract}

Key words. pulsars: general

\section{Introduction}

The polarization properties of more and more pulsars have been observed. They are important for our understanding of the pulsar emission process. The $S$-type PA curves of most pulsars are related to the rotation of the magnetic field plane where emission is generated, as described by the rotatingvector model. Recently, we noticed that if good quality measurements are available, almost all scatter broadened profiles have a flat PA curve in the trailing part without exception. Most previous work on scattering has concentrated on the total intensity profile (e.g. Rickett 1977; Lyne \& Graham-Smith 1998; Kuz'min \& Izvekova 1993; Bhat et al. 2003). Only a few just mention the possible effect of scattering on PA curves (Gil 1985; Wang et al. 2002) except for the early work by Komesaroff et al. (1972) on the Vela pulsar. In this research note, we discuss the scattering models and the effect of scattering on both total and polarized intensity profiles.

Scatter broadening is related to the geometry of the scattering disk (see Lyne \& Graham-Smith 1998). Compared to the radiation coming from the center of the disk, the extra delay $\tau$ of the scattered rays from a radius $r$ in a disk at distance $L$ should be $\tau=r^{2} / 2 c L$. Considering the probability of a scattered ray from $r$ to $r+\mathrm{d} r$, one finds an intensity variation with delay $t: I(t) \propto \exp \left(-2 c L / r^{2} t\right)$. Whenever the polarized radiation passes through the interstellar medium, as shown by Macquart \& Melrose (2000), all Stokes parameters should scintillate like the total intensity. Therefore, it is natural to assume that the scattering process works similarly on all Stokes parameters. We simulated the scattering effect on pulsar Stokes

Send offprint requests to: J. L. Han, e-mail: hjl@bao.ac.cn profiles and then checked the observational data of several pulsars. Using the observed high frequency mean pulse profiles without obvious scatter broadening as the intrinsic profiles, we can recover the low frequency observed profiles, including the flat PA curves, very well by convolving them with scattering models. We note that Komesaroff et al. (1972) had also shown that the broadened polarization profiles of the Vela pulsar (PSR B0833-45) are consistent with the scattering model.

\section{Scattering model and simulation}

The scattered pulse $y(t)$ can be derived by convolving the intrinsic pulse $x(t)$ with the response scattering function $g(t)$ :

$y(t)=\int x(\zeta) g(t-\zeta) \mathrm{d} \zeta$

The thin screen of interstellar medium between the pulsar and the observer can be modeled by a response function $g(t)=$ $\exp \left(-t / \tau_{\mathrm{sc}}\right.$ ) for $t>0$ (Kuz'min \& Izvekova 1993; Williamson 1972). The scattering time scale $\tau_{\text {sc }}$ can be related to the dispersion measure $(D M)$ and the observation wavelength $(\lambda)$ by an empirical relation

$\tau_{\mathrm{sc}}=4.5 \times 10^{-5} D M^{1.6} \times\left(1+3.1 \times 10^{-5} D M^{3}\right) \lambda^{4.4}$,

with $\tau_{\mathrm{sc}}$ in millisecond, $D M$ in $\mathrm{pc} \mathrm{cm}^{-3}$ and $\lambda$ in meter (see Mitra \& Ramachandran 2001).

The total intensity $(I)$ profile of the simulated pulse has a Gaussian shape, with linear polarization $(L)$ degree of $70 \%$ and a PA $(\psi)$ following the rotating-vector model; the Stokes parameters $Q$ and $U$ can be written as $Q=0.7 I \cos (2 \psi)$, $U=0.7 I \sin (2 \psi)$. The circular polarization $(V)$ is assumed to 


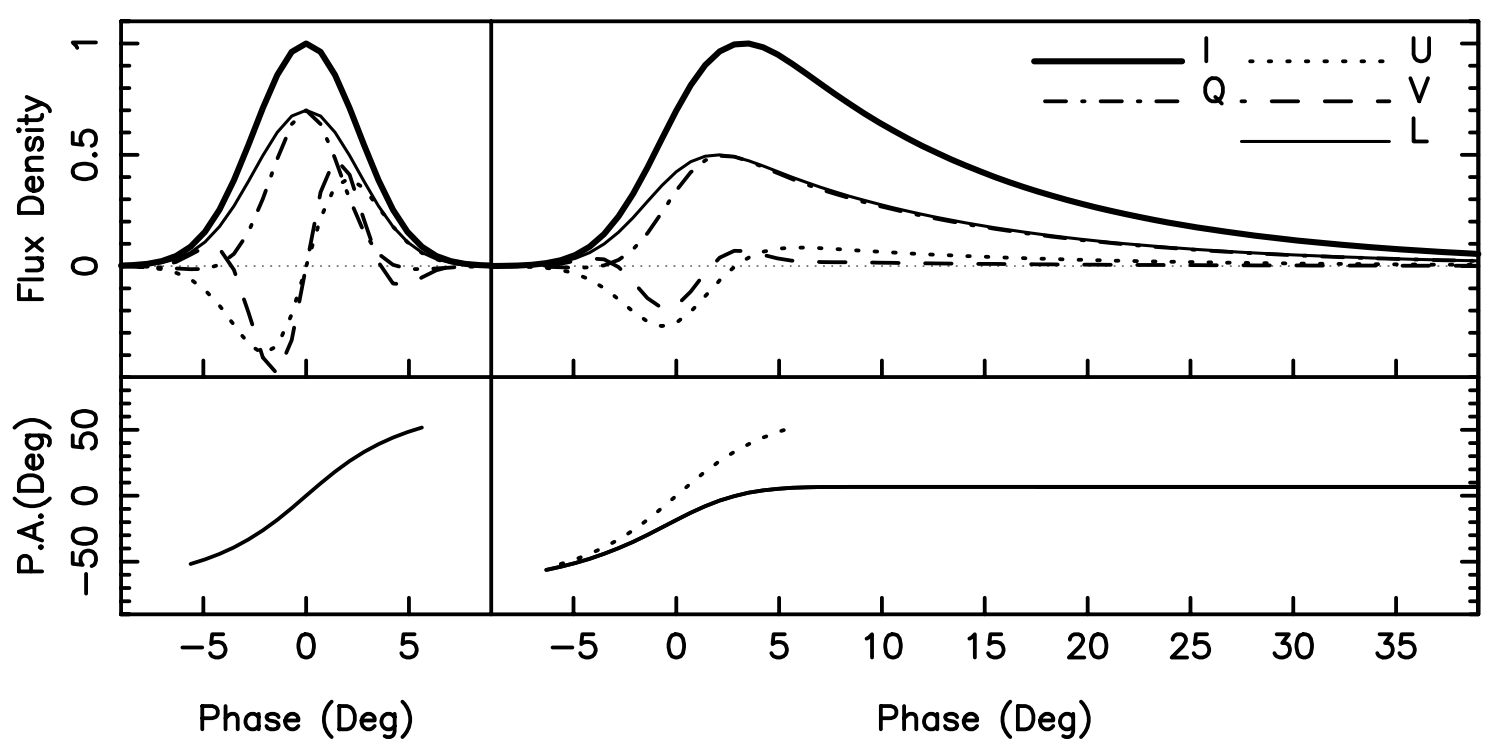

Fig. 1. The scattering effect of a thin screen on simulated Stokes polarization profiles. The simulated intrinsic $I, Q, U, V, L$ and PA are shown in the left panels, and the scattered ones in the right panels. The dotted line in the right-lower panel is the original PA. Obviously the PA curve of scattered profile is very flattened in the long pulse tail.

Table 1. Parameters of pulsar polarization profiles with obvious scattering at the lower frequency. Columns are pulsar name, period, dispersion measure $(D M)$, the higher and lower observation frequencies, sampling time at the lower frequency, the scattering time scale calculated by the empirical relation given in Eq. (2) $\left(\tau_{\mathrm{em}}\right)$, the time-scale of exponentially decaying tails $\left(\tau_{\mathrm{ex}}\right)$, the scattering time scale estimated from real data deconvolution $\left(\tau_{\mathrm{sc}}\right)$ and the corresponding scattering model used to fit the low frequency profiles.

\begin{tabular}{lclllccccc}
\hline \hline PSR & $P$ & $D M$ & Freq. & $\begin{array}{l}\text { Freq. } \\
\mathrm{GHz}\end{array}$ & $\begin{array}{c}T_{\mathrm{s}} \\
\mathrm{ms}\end{array}$ & $\begin{array}{c}\tau_{\mathrm{em}} \\
\mathrm{ms}\end{array}$ & $\begin{array}{c}\tau_{\mathrm{ex}} \\
\mathrm{ms}\end{array}$ & $\begin{array}{r}\tau_{\mathrm{sc}} \\
\mathrm{ms}\end{array}$ & Scattering model \\
\hline $1831-03$ & 0.6867 & 235.8 & 0.610 & 0.408 & 3.60 & 29.54 & 19.43 & 18.65 & thin screen \\
$1838-04$ & 0.1861 & 324.0 & 1.408 & 0.606 & 3.02 & 22.32 & 20.45 & $12.33 / 24.54$ & extended/thin \\
$1841-05$ & 0.2557 & 411.0 & 1.408 & 0.610 & 3.84 & 57.13 & 53.74 & 61.28 & thin screen \\
$1859+03$ & 0.6554 & 402.9 & 0.925 & 0.606 & 3.75 & 60.79 & 14.77 & 13.74 & thin screen \\
$1946+35$ & 0.7173 & 129.1 & 0.610 & 0.408 & 1.97 & 1.87 & 16.00 & 12.74 & thin screen \\
\hline
\end{tabular}

have a sense reversal (see the left panels in Fig. 1). If the scattering acts on all the Stokes parameters as described by Eq. (1), we can convolve $I, Q, U, V$ with the thin scattering model to get all scattered quantities. Simulation results are shown in Fig. 1. We can see in the right panels that the PA curve of the scattered profile is flattened and the circular polarization almost vanishes in the trailing part.

\section{Scattering effect on observed pulsar profiles}

To check the effect of scattering on polarization profiles, we downloaded high quality multifrequency polarization data of 5 pulsars (Gould \& Lyne 1998) available from EPN database ${ }^{1}$ (Lorimer et al. 1998), for which scatter broadening is very obvious in low frequency profiles (see Figs. 2-6).

The high frequency profiles without obvious scatter broadening were considered to be the intrinsic emitted pulse profiles for reference. The scattering time scales (Cols. 7-9 in Table 1) at lower frequencies can be either estimated using Eq. (2) in Col. 7 or derived by maximizing the correlation coefficients between the total intensity profile observed at lower

\footnotetext{
${ }^{1}$ http://www.mpifr-bonn.mpg.de/pulsar/data/
}

frequency and the profiles convolved from high frequency profiles (Table 1) with a thin screen model (Col. 9). In fact, it can also be estimated directly from the exponential tails (Col. 8). Using the scattering time scale from the cross-correlation (with an uncertainty of about $20 \%$ ), we then calculated each Stokes profile of 4 pulsars using the thin screen model. The convolved and observed profiles as shown in Fig. 2 and Figs. 4-6 are very consistent. We noticed that the convolved profiles of PSR B1838-04 at $0.606 \mathrm{GHz}$ using a thin screen model do not fit the observed ones (Fig. 3), so we tried a thick screen and an extended medium (Williamson 1972) for response functions. The extended medium model

$g(t)=\left(\frac{\pi^{5} \tau_{\mathrm{sc}}^{3}}{8 t^{5}}\right)^{1 / 2} \exp \left(-\frac{\pi^{2} \tau_{\mathrm{sc}}}{4 t}\right)$

does work very well.

The scattering time-scales we deconvolved from lower frequency data are consistent with earlier estimates if scaled with frequency, i.e. PSR B1831-03 in Ramachandran et al. (1997) and others in Taylor et al. (1993). While the estimate given by the empirical relation in Eq. (2) is a good description of the general tendency against $D M$, for a given $D M$ they can differ 

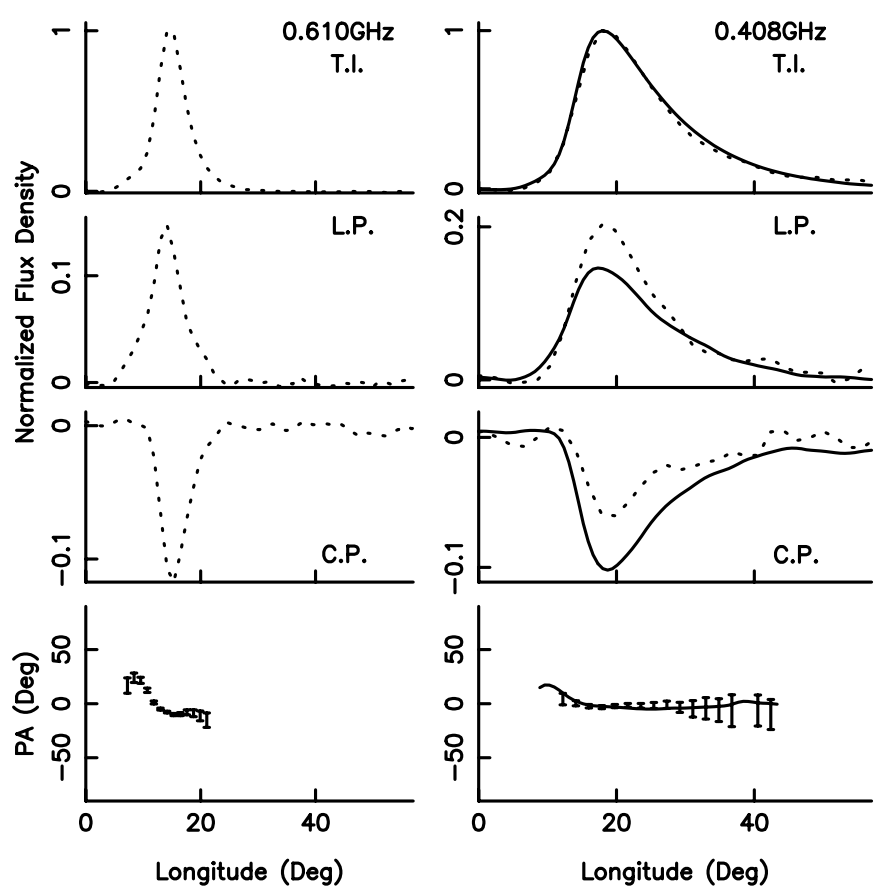

Fig. 2. Polarization profiles of PSR B1831-03. Total intensity (T.I.), linearly polarized intensity (L.P.), circular polarization (C.P.) and polarization angle (PA) are shown in different panels for two frequencies. All intensity profiles have been normalized to the peak of total intensity. The dotted lines are observed data. The errors of PA were given by $\sigma_{\psi}=\sqrt{Q^{2} \sigma_{U}^{2}+U^{2} \sigma_{Q}^{2}} /\left(2 L^{2}\right)$. The solid lines are profiles convolved by the highest frequency pulse profile with a thin scattering screen.
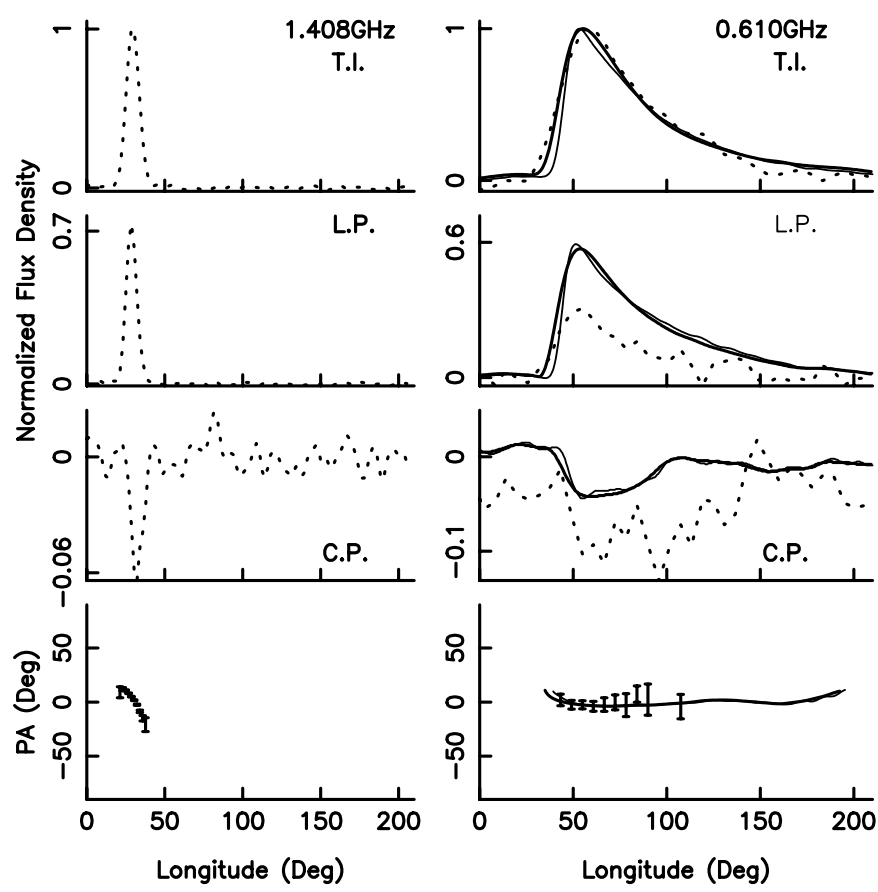

Fig. 3. The same as Fig. 2, but for PSR B1838-04 and convolved with an extended scattering medium (thick lines) and a thin model (thin lines).

by more than one magnitude (Mitra \& Ramachandran 2001; Ramachandran et al. 1997).
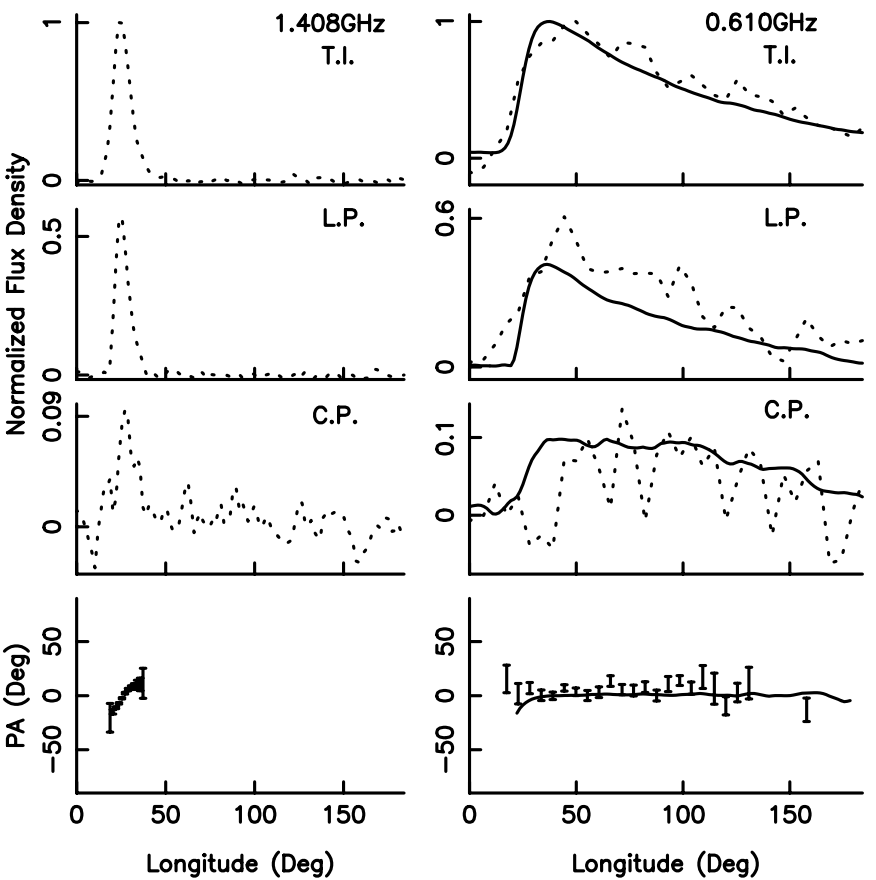

Fig. 4. The same as Fig. 2, but for PSR B1841-05.
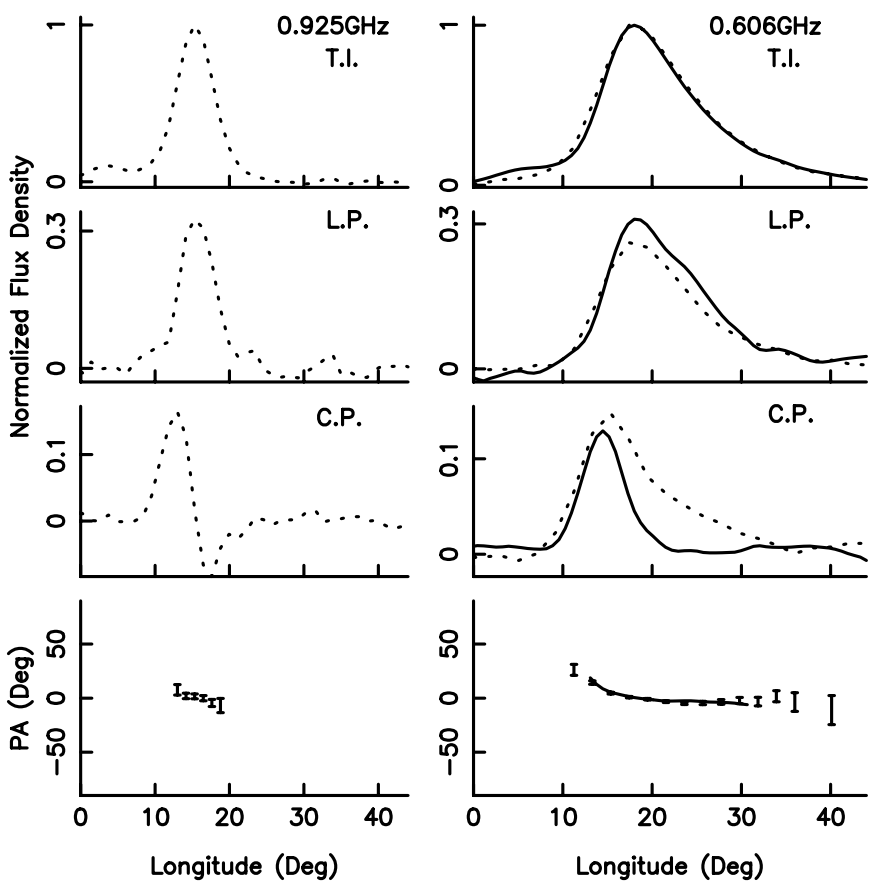

Fig. 5. The same as Fig. 2, but for PSR B1859+03.

Obviously when $\tau_{\mathrm{sc}}$ increases, the PA curves can be completely flattened irrespective of the original PA curve shape. In this case, the maximum gradient of the PA curve has been diminished and cannot be directly used to determine the geometry of the pulsar emission region (e.g. Wang et al. 2002). As we have tested, it is in principle possible to recover all Stokes profiles from the scattered ones as long as the a proper scattering model is used. However, one cannot be sure that the scattering material is of the thin-screen type, or extended, or in multi-thin-screen form. Consequently, the merit of 

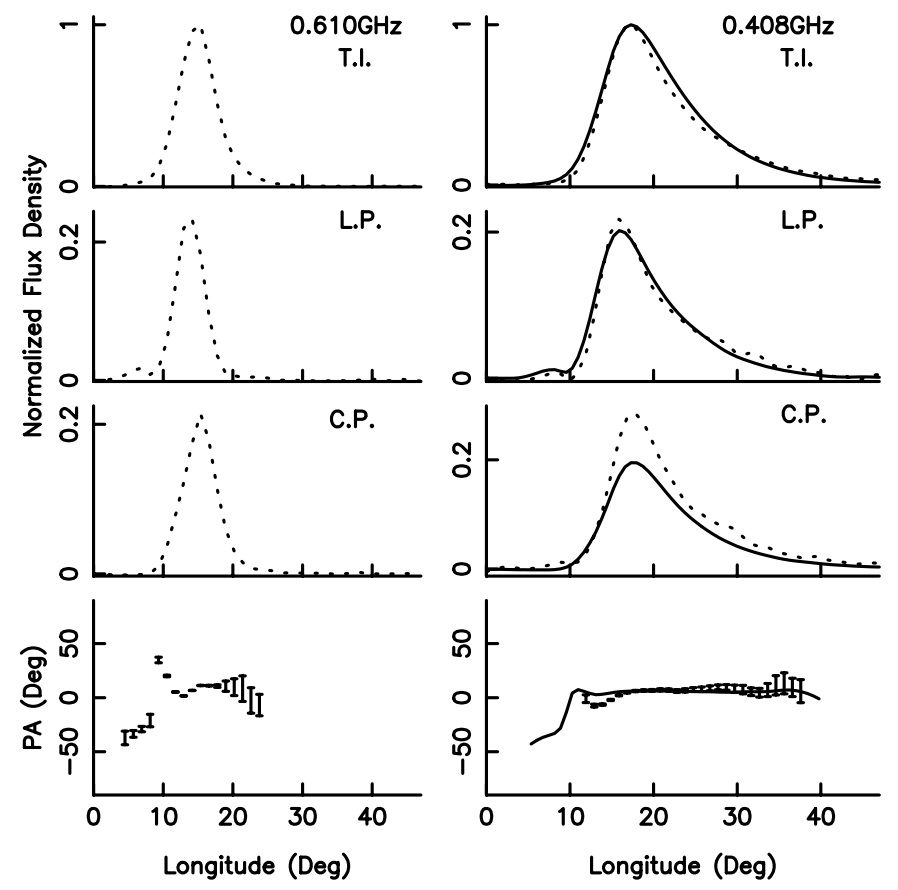

Fig. 6. The same as Fig. 2, but for PSR B1946+35.

deconvolved profiles is difficult to assess. For example, two possible results for PSR J1852+0031 from the two kinds of scattering medium given by Bhat et al. (2003) are both acceptable. It would be difficult to say which one is more likely to be the intrinsic one.

The recent Parkes multibeam Survey has discovered many pulsars with scatter broadened profiles (Manchester 2001). Polarization observations of some of them have shown the flattened PA curves in the trailing part (e.g. PSR J1730-3350 in Crawford et al. 2001). The leading part in fact cannot be used to determine the geometrical parameters of the pulsar, mainly because it has also been influenced by scattering (see simulation in Fig. 1). However, if the scattering model can be determined by comparing the scattered profile with one at higher frequency, then it is worth trying to recover the intrinsic Stokes profiles.

It is also worthy to note that not all flat PA curves are related to the scattering effect. For example, the flat PA curves of millisecond pulsars, e.g. PSR B1937+21 (Thorsett \& Stinebring 1990), at higher frequencies are obviously intrinsic, and probably not related to the scattering process.
From simulations, we also noticed that when the sense reversal appears in the intrinsic circular polarization profile, the scattering can largely weaken the circular polarization in the trailing part, as shown by PSR B1859+03 (Fig. 5). If there is no sense reversal, as in the other 4 pulsars shown, the singlehand circular polarization profiles are simply stretched.

\section{Conclusion}

We investigated the scattering effect on polarization profiles, especially position angle. The scattering can flatten the PA curves. Taking the high frequency profiles of 5 pulsars as intrinsic pulses, the observed low frequency scattered polarization profiles can be reproduced by convolving the high frequency observations with the scattering models.

Acknowledgements. We thank the referee, Prof. R. G. Strom, for his insightful suggestions and careful readings which improved the paper a lot. We are very grateful to X.H. Sun for carefully reading the manuscript. JLH is supported by the National Natural Science Foundation of China (19903003 and 10025313) and the National Key Basic Research Science Foundation of China (G19990754) as well as the partner group of MPIfR at NAOC.

\section{References}

Bhat, N. D. R., Cordes, J. M., \& Chatterjee, S. 2003, ApJ, 584, 782 Crawford, F., Manchester, R. N., \& Kaspi, V. M. 2001, AJ, 122, 2001 Gil, J. A. 1985, A\&A, 143, 443

Gould, D. M., \& Lyne, A. G. 1998, MNRAS, 301, 235

Komesaroff, M. M., Hamilton, P. A., \& Ables, J. G. 1972, Austr. J. Phys., 25, 759

Kuz'min, A. D., \& Izvekova, V. A. 1993, MNRAS, 260, 724

Lorimer, D. R., Jessner, A., Seiradakis, J. H., et al. 1998, A\&AS, 128, 541

Lyne, A. G., \& Graham-Smith, F. 1998, Pulsar Astronomy (Cambridge University Press)

Macquart, J.-P., \& Melrose, D. B. 2000, Phys. Rev. E, 62, 4177

Manchester, R. N. 2001, Ap\&SS, 278, 33

Mitra, D., \& Ramachandran, R. 2001, A\&A, 370, 586

Ramachandran, R., Mitra, D., Deshpanda, A. A., et al. 1997, MNRAS, 290, 260

Rickett, B. J. 1977, ARA\&A, 15, 479

Taylor, J. H., Manchester, R. N., \& Lyne, A. G. 1993, ApJS, 88, 529

Thorsett, S. E., \& Stinebring, D. R. 1990, ApJ, 361, 644

Wang, H. G., Xu, R. X., \& Qiao, G. J. 2002, ApJ, 578, 385

Williamson, I. P. 1972, MNRAS, 157, 55 